\title{
Source-receptor matrix calculation with a Lagrangian particle dispersion model in backward mode
}

\author{
P. Seibert and A. Frank \\ Institute of Meteorology and Physics, University of Natural Resources (BOKU), Wien, Austria \\ Received: 17 June 2003 - Published in Atmos. Chem. Phys. Discuss.: 29 August 2003 \\ Revised: 12 December 2003 - Accepted: 10 January 2004 - Published: 23 January 2004
}

\begin{abstract}
The possibility to calculate linear-source receptor relationships for the transport of atmospheric trace substances with a Lagrangian particle dispersion model (LPDM) running in backward mode is shown and presented with many tests and examples. This mode requires only minor modifications of the forward LPDM. The derivation includes the action of sources and of any first-order processes (transformation with prescribed rates, dry and wet deposition, radioactive decay, etc.). The backward mode is computationally advantageous if the number of receptors is less than the number of sources considered. The combination of an LPDM with the backward (adjoint) methodology is especially attractive for the application to point measurements, which can be handled without artificial numerical diffusion. Practical hints are provided for source-receptor calculations with different settings, both in forward and backward mode. The equivalence of forward and backward calculations is shown in simple tests for release and sampling of particles, pure wet deposition, pure convective redistribution and realistic transport over a short distance. Furthermore, an application example explaining measurements of Cs-137 in Stockholm as transport from areas contaminated heavily in the Chernobyl disaster is included.
\end{abstract}

\section{Introduction}

The source-receptor (s-r henceforth) relationship is an important concept in air quality modelling. It describes the sensitivity of a "receptor" element $y$ to a "source" $x$. The receptor could be, for example, the average concentration of a certain atmospheric trace substance in a given grid cell during a given time interval. It could also be a deposition value,

Correspondence to: $\mathrm{P}$. Seibert

(www.boku.ac.at/imp/envmet/seibert_mail.html) and instead of a grid volume average it could be the value at a measurement station. The source could be a point, area or volume source acting during a specified time interval in a specific location.

The s-r relationship can be linear or nonlinear. In the nonlinear case, it is defined by the partial derivative $\partial y / \partial x$ at a given state of the system. This paper deals with linear s-r relationships, because standard Lagrangian particle dispersion models cannot simulate nonlinear chemical reactions. However, all the other processes occurring during the atmospheric transport of trace substances are linear: advection, diffusion, convective mixing, dry and wet deposition, and radioactive decay. First-order chemical reactions, where the reaction rates can be prescribed, are also linear. In the linear case, the $\mathrm{s}-\mathrm{r}$ relationship reduces to the simple expression $y / x$. Thus, linear s-r relationships can be calculated easily with any dispersion model $\mathcal{M}: x \rightarrow y$.

In typical applications, there are many receptor elements and/or many sources. We will denote them by vectors $\boldsymbol{y}$ and $\boldsymbol{x}$, respectively. The $\mathrm{s}-\mathrm{r}$ relationships can be written as a s-r matrix (also abbreviated as SRM) $\boldsymbol{M}$ whose elements $m_{i l}$ are defined by

$m_{i l}=\frac{y_{l}}{x_{i}}$

In the case of gridded data, the temporal and all the three spatial dimensions are combined into each of the indices $(i=1, \ldots, I$ for the sources, and $l=1, \ldots, L$ for the receptors). This notation is in agreement with inverse modelling studies. In the remaining sections of this paper, receptors are specified as mixing ratios or concentrations and as such denoted with symbols $\chi$ and $c$. Sources are specified as mass flux densities or masses and denoted by $\dot{q}$ or $Q$, respectively. Thus, the s-r relationship can be denoted by $\partial \chi / \partial \dot{q}$ or similar expressions. 
The advantage of knowing $\boldsymbol{M}$ is that for a given source vector, the resulting receptor values can be obtained by a simple matrix-vector multiplication, avoiding the evaluation of the whole numerical model of the transport and dispersion processes. This is useful for scenario considerations. Furthermore, the SRM contains detailed budget information; it describes which receptors are affected by a specific source element (by the corresponding column of $\boldsymbol{M}$ ) and which sources are contributing to a specific receptor (by the row of $\boldsymbol{M}$ ) corresponding to the receptor). The SRM gives a quite comprehensive description of a transport and dispersion problem. Its determination is therefore also an important intermediate step for solving optimisation problems, such as the minimisation of environmental impact under defined abatement costs, or inverse modelling to determine sources from given measurements.

The determination of the SRM is much more costly in terms of computing than a single simulation with a given source configuration because it has to be either rerun for each source element, or (more efficiently but also requiring more computer memory) a separate species has to be tracked for each source element (in forward mode). In the case of $I>L$ (more source elements than receptor elements), receptor-oriented methods are more efficient than forward methods, and vice versa. These receptor-oriented methods are known as adjoint or backward methods. They need a number of simulations or species equal to the number of receptors (whereas forward methods need as many simulations or species to be tracked as there are sources). For the case of Eulerian models, many authors have pointed this out and applied adjoint Eulerian models for such tasks (e.g. Uliasz (1983), Uliasz and Pielke (1992), Robertson and Persson (1992), Giering (1999), Hourdin and Issartel (2000)). For Lagrangian particle models, Thomson (1987) and Flesch et al. (1995) have shown that the Lagrangian particle model is basically self-adjoint (except the sign of advection). Flesch et al. (1995) introduced backward integration of such a model as a tool to determine so-called footprints for measurements of micro-meteorological fluxes. Footprint modelling including the backward methodology has become an established tool in the micrometeorological community for interpretation of both fluxes and scalar quantities and the planning of measurements (Schmid, 2002; Kljun et al., 2002).

Seibert (2001) introduced this concept in air pollution modelling and for arbitrary volume sources. However, all these papers were limited to conservative tracers, without considering sinks or any other first-order processes.

The present papers shows that a Lagrangian particle dispersion model can be used in a backward-running, receptororiented mode to determine s-r relationships including any such first-order processes. The concept is valid for source and receptor volumes of arbitrary shape and location. We apply it to problems ranging from mesoscale to global. The derivation is followed by sample calculations illustrating practically the equivalence between forward and backwardmode calculations and an application example. Especially Test 3 and the associated Fig. 1 may serve to help understand the principle of s-r relationships and their calculation by forward or backward calculations.

Methods presented here have been integrated into the model Flexpart ${ }^{1}$ (Stohl et al., 1998), which was also used for the sample calculations. This model is presently used by about 15 groups all over the world and it is freely available. We feel therefore that it is not inappropriate to include some remarks on a more technical level which are related specifically to this model.

To avoid misunderstandings, we would like to point out that the Lagrangian particle dispersion model should not be confused with other types of Lagrangian models such as standard trajectory models or box models tied to such standard trajectories. The LPDM is a fully three-dimensional model including the mean horizontal and vertical wind and threedimensional turbulence.

\section{Theory}

\subsection{Forward simulations with an LPDM}

In the forward mode, particles are released in prescribed source regions, and transported with the mean and a stochastic turbulent velocity field. They carry a mass depending on source strength and particle release rate which can be altered by processes such as dry and wet deposition or decay. Gridded concentrations are calculated by summing up the masses of all the particles in a grid cell, and dividing the sum by the cell's volume. More refined formulations, used for example in the model Flexpart (Stohl et al., 1998) may apply kernels (Haan, 1999) to determine gridded and especially singlereceptor point concentrations.

In the backward mode, used for receptor-oriented modelling, the same formalism and computer model are applied, but the particle trajectories are integrated backward in time, using a negative time step. However, these particles are only a means to determine the trajectories and to probe the processes experienced by substances transported. Furthermore, the approach is based on mass mixing ratios rather than mass concentrations, as this is a conservative quantity.

\footnotetext{
${ }^{1}$ more information at http://www.forst.tu-muenchen.de/EXT/ LST/METEO/stohl/flexpart.html
} 
2.2 Lagrangian, receptor-oriented view of transport and dispersion

In the following, a mathematical description of the transport and dispersion process in the atmosphere including sources and linear processes (such as, e.g. deposition or decay) is given in a Lagrangian framework with a receptor-oriented view. The result is compared to the formalism employed in typical Lagrangian particle dispersion models.

We consider the individual change of the mass mixing ratio $\chi$ of an atmospheric trace substance. As we are in a Lagrangian frame of reference, there are no advective changes and $\chi$ is affected only by sources $\dot{q}$ or by any linear processes which are proportional to $\chi$. Nonlinear processes are not considered in this paper. The sources $\dot{q}$ are given as mass per volume and time. Thus,

$\frac{d \chi(t)}{d t}=\frac{1}{\rho(t)} \dot{q}(t)+\alpha(t) \chi(t)$

where $\alpha$ is the net decay or build-up rate "constant" and $t$ is time.

This ordinary differential equation can be solved, yielding the mixing ratio at time $t$ and location $\boldsymbol{r}^{*}$ :

$$
\begin{gathered}
\chi\left(\boldsymbol{r}^{*}, t\right)=\chi_{0} \exp \left(-\int_{0}^{t} \alpha\left[\boldsymbol{r}\left(t^{\prime}\right), t^{\prime}\right] d t^{\prime}\right)+ \\
\int_{0}^{t} \frac{\dot{q}\left[\boldsymbol{r}\left(t^{\prime}\right), t^{\prime}\right]}{\rho\left[\boldsymbol{r}\left(t^{\prime}\right), t^{\prime}\right]} \exp \left(-\int_{t^{\prime}}^{t} \alpha\left[\boldsymbol{r}\left(t^{\prime \prime}\right), t^{\prime \prime}\right] d t^{\prime \prime}\right) d t^{\prime}
\end{gathered}
$$

where $\left\{\boldsymbol{r}\left(t^{\prime}\right), t^{\prime} \leq t\right\}$ is the back trajectory arriving at point $\boldsymbol{r}^{*}$ at time $t$, and $\chi_{0}$ is the initial mixing ratio (background) at $t=0$.

Let us write as an abbreviation

$$
p\left(t^{\prime}\right)=\exp \left(-\int_{t^{\prime}}^{t} \alpha\left[\boldsymbol{r}\left(t^{\prime \prime}\right), t^{\prime \prime}\right] d t^{\prime \prime}\right)
$$

and call it the transmission function because it determines the fraction of material which is transmitted along a trajectory to the receptor. Let us further write in abbreviated form $\dot{q}\left(t^{\prime}\right)$ and $\rho\left(t^{\prime}\right)$ with the meaning that it denotes $\dot{q}$ and $\rho$ along the trajectory as explained above. Equation (2) then reads

$$
\chi\left(\boldsymbol{r}^{*}, t\right)=\chi_{0} p(0)+\int_{0}^{t} \frac{\dot{q}\left(t^{\prime}\right)}{\rho\left(t^{\prime}\right)} p\left(t^{\prime}\right) d t^{\prime} .
$$

Equations (2) and (4) are valid for instantaneous mixing ratios, affected by turbulent fluctuations. The mean mixing ratios should be obtained as ensemble averages. In practical applications, measured concentrations at monitoring stations represent nearly a point in space but an average in time.

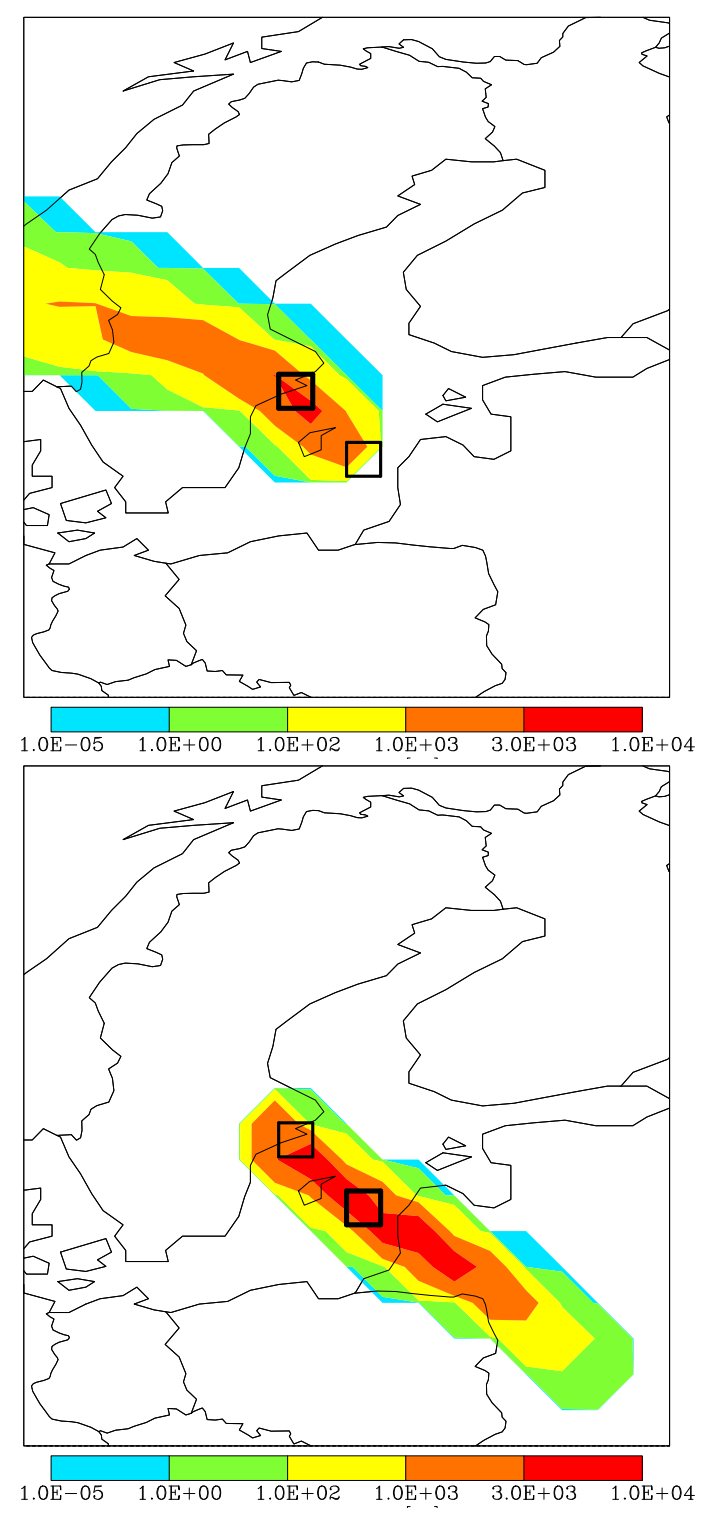

Fig. 1. Example of the forward, source-oriented mode (top) and the backward, receptor-oriented mode (bottom) calculations performed for Test 3: realistic short-range transport of a conservative tracer. Calculations were performed for 11 October 2000 over the Baltic Sea and its surroundings (coast lines and borders (as of 1986) are shown by thin lines). The light square indicates the release areas in each mode. Sampling is performed on the whole grid, but only the sample of the bold square can be used for comparison of the forward-mode and backward-mode calculation of the s-r relationship. The length of each square is $1^{\circ}$. The parameter shown is the s-r relationship [s], calculated with the mode represented by green squares in Fig. 2.

Thus, we need to average $\chi$ in time. If the averaging time exceeds well the time scale of the turbulent fluctuations of $\chi$ (and it does so in trace substance monitoring), the temporal average of the instantaneous values is the same as the 
temporal average of the respective ensemble means $(\bar{\chi}=\overline{\langle\chi\rangle}$, where the overbar indicates temporal averaging and \langle\rangle the ensemble averaging). Similar considerations hold for aircraft measurements which are typically line integrals or satellite measurements representing volume integrals. For the mean (time-integrated) mixing ratio $\bar{\chi}$ we obtain

$$
\bar{\chi}\left(t_{1}, t_{2}\right)=\frac{1}{t_{2}-t_{1}} \int_{t_{1}}^{t_{2}} \chi\left(\boldsymbol{r}^{*}, t\right) d t=
$$$$
\frac{1}{t_{2}-t_{1}} \int_{t_{1}}^{t_{2}}\left(\chi_{0}(t) p(t, 0)+\int_{0}^{t} \frac{\dot{q}\left(t, t^{\prime}\right) p\left(t, t^{\prime}\right)}{\rho\left(t, t^{\prime}\right)} d t^{\prime}\right) d t,
$$

where a second time variable $t$ appears in $\dot{q}, \rho$, and $p$ to denote trajectories arriving at different times $t$.

We now introduce a discretisation. Arrival time $t$ is discretised into $J$ time slots each represented by one back trajectory. The trajectories arrive at equal intervals between $t_{1}$ and $t_{2}$ and are designated by index $j$. Space is gridded with index $i$. Furthermore, we need a discretisation of $t^{\prime}$, denoted by the index $n$. This leads to

$\bar{\chi} \approx \overline{\chi_{0} p(0)}+\frac{1}{J} \sum_{j} \sum_{i} \sum_{n}\left(\frac{\dot{q}_{i n}}{\rho_{i n}} p_{j n} \Delta t_{i j n}^{\prime}\right)$

with $\Delta t_{i j n}^{\prime}$ being the residence time of trajectory $j$ in the spatio-temporal grid cell $(i, n)$. Note that the integration along the trajectory (a line integral in the joint time-space dimension) has been replaced by a double sum, one over time and one over space, with the residence time being not only the discrete representation of $d t$ but also the indicator of the trajectory movement. As the source function $\dot{q} / \rho$ depends only on the time when the back trajectory passes but not on the time when it arrives at the receptor, Eq. (6) can be rearranged to (writing $=$ instead of $\approx$ for simplicity)

$\bar{\chi}=\overline{\chi_{0} p(0)}+\sum_{i} \sum_{n}\left[\frac{\dot{q}_{i n}}{\rho_{i n}} \frac{1}{J} \sum_{j}\left(p_{j n} \Delta t_{i j n}^{\prime}\right)\right]$.

We are now able to calculate the s-r relationship:

$$
\frac{\partial \bar{\chi}}{\partial \dot{q}_{i n}}=\frac{1}{J} \sum_{j} \frac{p_{j n} \Delta t_{i j n}^{\prime}}{\rho_{i n}} .
$$

In the case of $\alpha=0$ and thus $p=1$, the s-r relationship for a source expressed as mass mixing ratio $(\dot{q} / \rho)$ is the average residence time of the back trajectories in the grid cell of the source under consideration, in agreement with the earlier derivation for this specific case (Seibert, 2001). In the general case, the residence times are to be "corrected" with the transmission function $p$.
2.3 Computation of transmission-corrected residence times with a Lagrangian particle dispersion model

The transmission-corrected residence times, yielding the s-r relationships according to Eq. (8), can be calculated easily with a Lagrangian particle dispersion model running backward in time. As discussed in in Sect. 2.1, regular LPDMs generate particles at source locations, follow their forward trajectories, and let different processes such as deposition act on the mass attached to the computational particle. By reversing the sign of the advection, the trajectories can easily be calculated backward. The LPDM Flexpart (Stohl et al., 1998) has this capability already built in. It required a few other modifications in the code, allowing input and output to occur in reverse temporal direction, etc. The first-order processes that have been summed up in the function $\alpha$ remain the same as in a forward calculation, in the sense that the mass will be, e.g. reduced by decay whether going backward or forward. Due to the linear nature of the term $\alpha \chi$, the direction of the time-stepping does not matter. If the backward calculation reverses the sign of the time step instead of the advection velocities (as does Flexpart), the integration of processes related to $\alpha$ must be carried out with the absolute value of the time step.

Turbulence is represented in LPDMs by a stochastic process with a prescribed pdf for the turbulent wind components (or their random parts, if a model with autocorrelation is used). Let $P\left(v_{i}\right)$ be the probability density function for the velocity component $v_{i}$. In order to calculate back trajectories, this probability must be replaced by $P\left(-v_{i}\right)$ (Flesch et al., 1995). If only a symmetric pdf is implemented, for example a Gaussian distribution as in Flexpart, no code modification is required. An integration with a negative time step will give the correct backward trajectories. However, the cloud convection scheme in Flexpart has different probabilities for upward and downward movements and thus needed to be adapted (see Sect. 2.6).

The statistics of the turbulence do not depend on the direction of a trajectory. In mathematical terms, this means that the Lagrangian equation of transport in a turbulent fluid, in the absence of asymmetric random motions as discussed above, is self-adjoint with the exception of a change of sign in the mean-flow advection (Flesch et al., 1995).

Let us now see how the standard output of a model like Flexpart can be used to extract these transmission-corrected residence times. Flexpart writes out mass concentrations $\bar{c}$, averaged over a grid element (index $i$ ) and a time interval $\Delta T$ (index $n$ ). They are computed as

$$
\bar{c}_{i n}=\frac{1}{V_{i} N^{*}} \sum_{n^{*}=n+1}^{n+N^{*}} \sum_{j=1}^{J} \mu_{j n^{*}} f_{i j n^{*}},
$$


where $N^{*}$ is the number of samples taken within $\Delta T$, and the time-dependent particle mass $\mu_{j n^{*}}$ is

$\mu_{j n^{*}}=\mu_{0} p_{j n^{*}}$

with $\mu_{0}$ being the initial mass associated with a particle (which is a constant for each release in Flexpart). $f_{i j n^{*}}$ is a function that determines if and how much a particle contributes to a given grid cell. In the simplest case, $f_{i j n^{*}}$ would be 1 if trajectory $j$ is in grid cell $i$ at time step $n^{*}$ and 0 otherwise; in Flexpart, it is used with a kernel formulation which distributes the mass over four grid elements as a measure to counteract the numerical errors due to the limited number of trajectories. Combining Eqs. (9) and (10), we obtain for the LPDM output:

$\bar{c}_{i n}=\frac{\mu_{0}}{V_{i} N^{*}} \sum_{n^{*}=n+1}^{n+N^{*}} \sum_{j=1}^{J} p_{j n^{*}} f_{i j n^{*}}$

The calculation of the s-r relationship with the LPDM requires, of course, that the temporal discretisation of the source as introduced in the previous section matches the time intervals used for averaging the output in the LPDM. For a numerical representation, we can adopt the same strategy as in Flexpart and divide the time interval of interest into $N^{*}$ small time slices, using the same function $f$ for each of these intervals. Thus, Eq. (8) becomes

$$
\frac{\partial \bar{\chi}}{\partial \dot{q}_{i n}}=\frac{\Delta T}{N^{*}} \sum_{n^{*}=n+1}^{n+N^{*}} \frac{1}{J} \sum_{j=1}^{J} \frac{p_{j n^{*}} f_{i j n^{*}}}{\rho_{i n^{*}}} .
$$

Considering that $J$ is independent of $n^{*}$, we may write

$$
\frac{\partial \bar{\chi}}{\partial \dot{q}_{i n}}=\frac{V_{i} \Delta T}{J \mu_{0}} \overline{\left(\frac{c_{i n}}{\rho_{\text {in }}}\right)}=\frac{V_{i} \Delta T}{\mu_{t o t}} \overline{\left(\frac{c_{i n}}{\rho_{\text {in }}}\right)}
$$

with $\mu_{t o t}$ as abbreviation for the total initial mass released in the time interval between $t_{1}$ and $t_{2} . \overline{\left(c_{i n} / \rho_{i n}\right)}$ differs from the standard output of the LPDM as given in Eq. (11), $\bar{c}_{i n}$, only in the density by which the contributions of the single particles in an output grid cell have to be divided. This division is necessary because we have expressed the source in mass units. If, instead, the source would be specified as alteration rate of the mixing ratio, the standard output $\bar{c}_{i n}$ could be used directly. Note that in this case the dimension of the r.h.s. is time. In any case, it is obvious that the standard LPDM provides a way to obtain $\mathrm{s}-\mathrm{r}$ relationships by running it backward in time with very few modifications.

A key assumption made to arrive at this result is that particles carry mixing ratios rather than masses. This is equivalent to the change from $\nabla \cdot \rho \chi \boldsymbol{v}$ to $\boldsymbol{v} \cdot \nabla \rho \chi$ between forward and adjoint Eulerian transport models (Elbern and Schmidt,
Table 1. Implementation scheme for different concentration units in an LPDM. "mass" refers to mass units $\left(\mathrm{kg} \mathrm{m}^{-3} \mathrm{~s}^{-1}\right.$ for the source, $\mathrm{kg} \mathrm{m}^{-3}$ for the receptor), "mix" to mixing ratio units $\left(\mathrm{s}^{-1}\right.$ for the source, dimensionless for the receptor). Given are in the column "release" the factor with which the initial mass of a particle has to be multiplied, and in the column "sampling" the factor to multiply particles' masses with at the moment of sampling. Note that in backward simulations the release takes place at the receptor and the sampling at the source. The respective units of the s-r relationship are given in the last column. The first value (C:) is for sources given as rates per time (and volume in the case of mass), the second one (I:) for instantaneous local sources (for example, a mass in units of

\begin{tabular}{|c|c|c|c|c|c|}
\hline source & receptor & mode & release & sampling & $\mathrm{s}-\mathrm{r}$ unit \\
\hline \multirow[b]{2}{*}{ mass } & \multirow[b]{2}{*}{ mass } & fwd & 1 & 1 & $\mathrm{C}: \mathrm{s}$ \\
\hline & & bwd & $\rho$ & $\rho^{-1}$ & $\left\{\mathrm{I}: \mathrm{m}^{-3}\right.$ \\
\hline \multirow{2}{*}{ mass } & \multirow{2}{*}{$\operatorname{mix}$} & fwd & 1 & $\rho^{-1}$ & $\mathrm{C}: \mathrm{kg}^{-1} \mathrm{~m}^{3} \mathrm{~s}$ \\
\hline & & bwd & 1 & $\rho^{-1}$ & $\mathrm{I}: \mathrm{kg}^{-1}$ \\
\hline \multirow{2}{*}{$\operatorname{mix}$} & \multirow{2}{*}{ mass } & fwd & $\rho$ & 1 & $\mathrm{C}: \mathrm{kg} \mathrm{m}^{-3} \mathrm{~s}$ \\
\hline & & bwd & $\rho$ & 1 & $\mathrm{I}: \mathrm{kg} \mathrm{m}^{-3}$ \\
\hline \multirow{2}{*}{$\operatorname{mix}$} & \multirow{2}{*}{$\operatorname{mix}$} & fwd & $\rho$ & $\rho^{-1}$ & S $\mathrm{C}: \mathrm{s}$ \\
\hline & & bwd & 1 & 1 & $\{\mathrm{I}: 1$ \\
\hline
\end{tabular}
$\mathrm{kg}$ ). They are the same for forward and backward calculations!

1999). At both 'ends' of a simulation, at the source and at the receptor, mass units may be desired. This is true for forward as well as for backward simulations. The conversion between both forms is accomplished by multiplication or division with the local air density. As density can vary within a source or receptor volume, this conversion is best done particle-wise. Work on this paper and associated tests as well as ongoing practical applications have shown that keeping maximum flexibility in an LPDM code with respect to mixing ratio versus mass units is desirable. Table 1 indicates how to set up a model for all the possible combinations. However, additional scalings are needed if the s-r relationship is to be derived from forward calculations and defined in the same way as done here (see Eq. 18).

\subsection{Practical considerations}

For gases, volume mixing ratios are a more popular unit than mass mixing ratios. The conversion is simple: multiplication with the ratio of the molecular mass of air to that of the gaseous trace substance or vice versa.

Another widespread practice is to report mass concentrations at standard temperature and pressure (STP). In this case, Table 1 can be applied with local air density $\rho$ replaced by the air density at STP. As this is a constant, it is possible, for example, to do a backward simulation for receptor units of mixing ratio, and to divide the resulting s-r relationship by $\rho_{\text {STP }}$ later in order to use it in conjunction with measurements given in STP concentrations. 
Another issue is that often we would be interested in an area source at ground level. To accomplish this, let us consider any area $A$ and a vertical layer adjacent to the ground of thickness $d$ as the source volume. Diluted over this layer, an area source $\dot{q}_{A}$ (in $\mathrm{kg} \mathrm{m}^{-2} \mathrm{~s}^{-1}$ ) would correspond to a volume source (in $\mathrm{kg} \mathrm{m}^{-3} \mathrm{~s}^{-1}$ ) $\dot{q}=\dot{q}_{A} d^{-1}$. Thus,

$$
\frac{\partial \bar{\chi}}{\partial \dot{q}_{A}}=\frac{1}{d} \frac{\partial \bar{\chi}}{\partial \dot{q}}
$$

Obviously, as $\partial \bar{\chi} / \partial \dot{q}_{A}$ contains the (density-weighted) residence time in the layer, it will, for well-mixed conditions and a layer not too deep, be proportional to $d$, so that $\partial \bar{\chi} / \partial \dot{q}_{A}$ is independent of $d$. The condition of being well-mixed is usually fulfilled within the boundary layer after some travel distance, except for a strongly depositing substance under stable conditions. To obtain good numerical stability, one should not choose the depth of the lowest layer too small, and except for the above-mentioned circumstances it can be chosen to be the typical minimum mixing height expected in the model. It is also interesting to note that the residence time in this layer of a single trajectory being reflected on the ground with vertical velocity $w$ is $2 d / w$. Thus, the s-r relationship for a conservative tracer $(p=1)$ and an area source is

$$
\frac{\partial \overline{\rho \chi}}{\partial \dot{q}_{A}}=\frac{1}{J d} \sum_{j=1}^{J} \frac{2 d f_{j}}{w_{j}}=2 \frac{J^{*}}{J} \overline{w^{-1}}
$$

twice the fraction of trajectories impinging on the ground weighted with the inverse of their vertical velocity, a conclusion already drawn by Flesch et al. (1995).

To complete the practical formulae, the s-r relationship for a source in $\mathrm{kg} \mathrm{s}^{-1}$ (point source, one-grid cell source), having units of $\mathrm{s} \mathrm{kg}^{-1}$, would be

$$
\frac{\partial \bar{\chi}}{V_{i} \partial \dot{q}_{i n}}=\frac{\Delta T}{\mu_{t o t}} \overline{\left(\frac{c_{i n}}{\rho_{i n}}\right)},
$$

and for an instantaneous point source (or the total source for a given time interval) in $\mathrm{kg}$, having units of $\mathrm{kg}^{-1}$,

$$
\frac{\partial \bar{\chi}}{\Delta T V_{i} \partial Q_{i n}}=\frac{1}{\mu_{t o t}} \overline{\left(\frac{c_{i n}}{\rho_{i n}}\right)} .
$$

Instead of expressing ambient concentrations and sources in kilogramme, one can always substitute that by Becquerel if radioactivity is under consideration.

Flexpart and other LPDMs allow not only to calculate gridded concentrations but also concentrations at point receptors, by means of suitable kernels sampling particles in a certain neighbourhood of the receptor. This can be used to obtain s-r relationships for point sources if the (candidate) source locations are known.
To convert gridded concentrations resulting from standard forward simulations into $s-r$ relationships with the dimension of time, a transformation is necessary. For source and receptor both in mass units, it reads as follows (for other cases, factors as indicated in Table 1 need to applied):

$$
\frac{\partial \bar{\chi}}{\partial \dot{q}_{i n}}=\frac{\Delta T_{s} V_{s}}{\mu_{t o t}} \bar{c},
$$

where $\Delta T_{S}$ is the time during which the (constant!) source is acting, $V_{s}$ is its volume and $\bar{c}$ is the concentration in a grid cell as delivered by the forward run. For Flexpart, the above value must be multiplied with the factor $10^{-12}$, because results are scaled by that factor.

\subsection{Dry and wet deposition}

Source-receptor relationships are also of interest with respect to receptors representing accumulated deposition. The deposition fluxes are obtained by linear operators on the the concentration field (in an LPDM, on the masses of particles). Therefore it is easy to extend the methods presented to this case. In an efficient implementation, pseudo-sources with strengths varying as a function of time and space, proportional to the deposition velocity or wash-out coefficients, would be used. This has not yet been implemented in Flexpart.

\subsection{Convection}

Most Lagrangian particle dispersion models do not simulate the effects of moist (i.e., cloud) convection. However, a parameterisation for convective transports has been introduced recently into Flexpart (Seibert et al., 2002). The scheme redistributes particles from their initial level to a randomly chosen destination level. The transition probabilities are given in discrete form in a so-called redistribution matrix which is calculated from the temperature and humidity profiles. Because convection manifests as concentrated updrafts with a high vertical velocity and weak compensating subsidence occupying a larger area, the redistribution matrix is not symmetric. In a backward run, particles must be redistributed from the destination level back to the initial level. Thus, the transposed redistribution matrix has to be used. This means that the probability of a particle of having arrived at its present level from another level is considered.

Another issue which requires attention is the part of the scheme which calculates the redistribution matrix. A one-dimensional convection model (Emanuel and ŽivkovićRothman, 1999), designed for use in a prognostic meteorological model, is run for each grid column containing particles. The model is integrated forward in time for a few hours, calculating Eulerian tracer tendencies due to the action of 
convection, from which the redistribution matrix is derived. Tendencies of the grid-scale temperature and humidity (taken from the meteorological input to Flexpart) enter this simulation, and these tendencies must be forward in time, as the convection submodel must always run forward in time, even in an LPDM backward simulation. The convection subroutines are called only once for each set of meteorological input fields, thus typically every 3 or $6 \mathrm{~h}$, in the middle of the time between the two fields (this has been changed in Flexpart recently). If a sampling or release period is not distributed symmetrically around the time when convection is calculated, differences between forward and backward mode modes will arise. They are an unavoidable consequence of the coarse temporal discretisation for the convection, which, however, is necessary because the convection simulation is so time-consuming.

\section{Test calculations}

Source-receptor relationships have been calculated both in forward and backward mode for a number of test cases. The purpose of these test calculations was to illustrate the concept and to have empirical support for the correctness of the derivations presented in the first part of this paper. During this work it turned out that these tests - comparison between forward and backward simulations as well as calculations for which the result can be analytically derived - is also a very useful validation check for a model. In Flexpart, we found a few bugs and model limitations in this process, mainly related to the numerical implementation of wet scavenging and to the release and sampling of particles. Most of these bugs would not affect standard applications in a significant way.

The tests are described here in the order of increasing complexity. They were carried out in final form with Flexpart Version 5; unless otherwise mentioned, bugs and limitations found were corrected for the tests. The next version of Flexpart will include all the improvements worked out during these tests. More details will be provided through the Flexpart home page on the world-wide web (cf. Footnote 1).

Presently, particles are released in standard forward mode of Flexpart equally distributed throughout the vertical extension of the source. It would be more realistic to release them with the same profile as air density, but again, as long as the layer is not too deep, the difference is not a big one.

\subsection{Test 1: Release and sampling}

In this test, particles representing a conservative tracer are released during one day in a certain volume which is at the same time the sampling volume. It was chosen to be $1^{\circ} \times 1^{\circ} \times 500 \mathrm{~m}$ large. All velocities (mean wind and turbu-
Table 2. Results of release and sampling test.

\begin{tabular}{lc}
\hline type of calculation & s-r relationship [s] \\
\hline analytical & 43,200 \\
forward & 43,233 \\
backward & 43,233 \\
\hline Number of particles: 1000 & \\
Synchronisation time interval: 300 s & \\
\hline
\end{tabular}

lence) are set to zero, and the sampling kernel (attributing the mass of each particle to the four surrounding grid cells) was switched off so that particles are attributed completely to the cell where they are located. Thus, the only processes present in the model were release and sampling of particles, and any errors are due to the discretisation of these processes. (Therefore we can work with a relative small number of particles.) The sampling took place during the same $24 \mathrm{~h}$ in which the source was active. Thus, the analytical s-r relationship is the mean residence time of the particles in the volume, 43200 s. Test results and some additional characteristics are reported in Table 2. There is no difference between forward and backward mode. The small difference (less than 1 per mille) between numerical and analytical result is caused by an inaccurate temporal discretisation of release and sampling of particles, affecting the first and last intervals only.

\subsection{Test 2: Wet scavenging}

This test is the same as Test 1, except that a strong wet scavenging is switched on with a prescribed, linearly varying precipitation rate. In the available versions of Flexpart, wet scavenging acts on all particles regardless of their height. Thus, the true s-r relationship could be calculated analytically. Scavenging is implemented in Flexpart as a scavenging rate which is a nonlinear function of the precipitation intensity (Stohl and Seibert, 2002). Furthermore, tests were conducted for stationary precipitation fields with convective precipitation only, large-scale precipitation only, or mixed precipitation, all with a total cloud cover of 1 . In all these tests, differences were at most a few seconds (Table 3 ).

\subsection{Test 3: Realistic scenario for short-distance transport}

In this test, particles were released in the same grid volume as in the previous tests, however, they were sampled in another grid volume two degrees to the south-east, during the same $24 \mathrm{~h}$ as the release. The co-ordinates of the grid centres were $57^{\circ} \mathrm{N}, 20^{\circ} \mathrm{E}$ and $59^{\circ} \mathrm{N}, 18^{\circ} \mathrm{E}$. Real meteorological fields (ECMWF analysis, $1^{\circ}$ horizontal resolution, 60 layers, every $3 \mathrm{~h}$ ) were used as input, and three different types of tracers were simulated: conservative, short-lived ("radioactive noble gas", half-life $0.5 \mathrm{~d}$ ), and a tracer subject to wet 


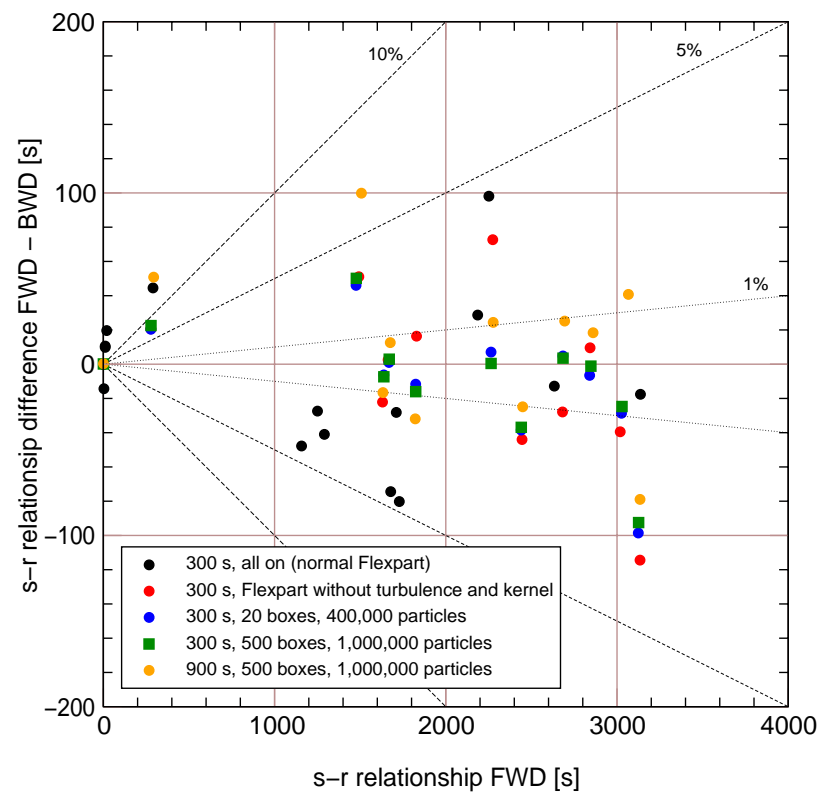

Fig. 2. Scatter plot of s-r relationships [s] for the realistic shortrange transport of a conservative tracer (Test 3 ). The differences between forward and backward calculation results are plotted against the forward results. Each dot represents one day. Dashed lines indicate relative deviations between the two calculation modes. The calculations were carried out with different time steps and particle numbers (partly released in sub-boxes of the source for more homogeneous initial distribution) as indicated in the insert. All calculations except the "normal Flexpart" are without turbulence and kernel and, unless otherwise indicated, with 40000 particles.

scavenging (“aerosol”, scavenging properties as in Test 2). This test was carried out for the days 1-20 October 2000. Convection was switched off (note that this refers only to vertical redistribution through moist convection, convective precipitation is taken from ECMWF analysis and is always considered). In this case, fluctuations of the concentrations occur as a result of the stochastic nature of the LPDM.

Figure 1 illustrates the test as well as the forward and backward calculations modes for the s-r relationship in general. In the forward mode, particles are released from the area of the light square, which is the source in the s-r relationship considered in this test, and tracked as they are transported by the atmospheric flow. The difference of this mode to regular dispersion modelling is only that results have been scaled according to Eq. (18). In the backward mode, particles are released from the area of the receptor in the s-r relationship (which is marked by the bold square in the forward-mode picture and the light square in the backward-mode picture) and tracked backward in time, to their regions of origin. In each calculation mode, only one sampling volume, indicated by the bold square, may be compared with the other mode.
Table 3. Results of wet scavenging test.

\begin{tabular}{lc}
\hline type of calculation & s-r relationship [s] \\
\hline analytical & 2668 \\
forward & 2666 \\
backward & 2664 \\
\hline Number of particles: 1000 & \\
Synchronisation time interval: $300 \mathrm{~s}$ & \\
Scavenging parameters: $a=2 \times 10^{-4}, b=0.8 \mathrm{~mm}^{-1} \mathrm{~h}$ \\
Precipitation rate $\left[\mathrm{mm} \mathrm{h}^{-1}\right.$ ]: $1.9+0.1 \mathrm{INT}(\mathrm{time} / 300 \mathrm{~s})$ \\
\hline
\end{tabular}

Figure 2 compares the s-r relationships calculated in forward and backward mode with different set-ups of the model. We notice that stochastic effects of turbulence produce deviations between both calculation modes. In one set-up, all turbulent components were set to zero. However, even then there are deviations. It was found that they can be diminished by using a large number of particles, and ensuring a homogeneous initial distribution by dividing the source into separate boxes. However, even then some deviations remain, and they decrease when shorter time steps are used. They are an expression of the inherent uncertainties of the trajectory calculation caused by the need to interpolate wind fields to particle positions.

This was corroborated by further tests. With artificial, constant wind fields no deviations occurred. One-dimensional trajectories were calculated with the numerical scheme of Flexpart in an analytical, nonlinear wind field. In the first case, velocities were calculated directly from the analytical formula at each position and in the second case they were interpolated from values at a regular grid. For time steps going towards zero, errors were found to go to zero as expected only in the first case, but not in the second one.

This is a further example that the forward-backward comparison is a useful model test in general, and it might even be used as to estimate the total interpolation error in the results.

No differences in behaviour, as far as equality between forward and backward simulations is concerned, were found between species.

\subsection{Test 4: Idealised convective redistribution}

An idealised test was devised for the cloud convection scheme, assuming complete mixing through moist convection. The redistribution matrix for the convection was defined analytically so that it would lead to complete vertical mixing within one step. The vertical levels used in this matrix were ECMWF levels from a real case, and 50000 particles were used. Tests were conducted with instantaneous, local sources and results are scaled to be valid for a one square-metre column. This test serves not only as a test for the backward 

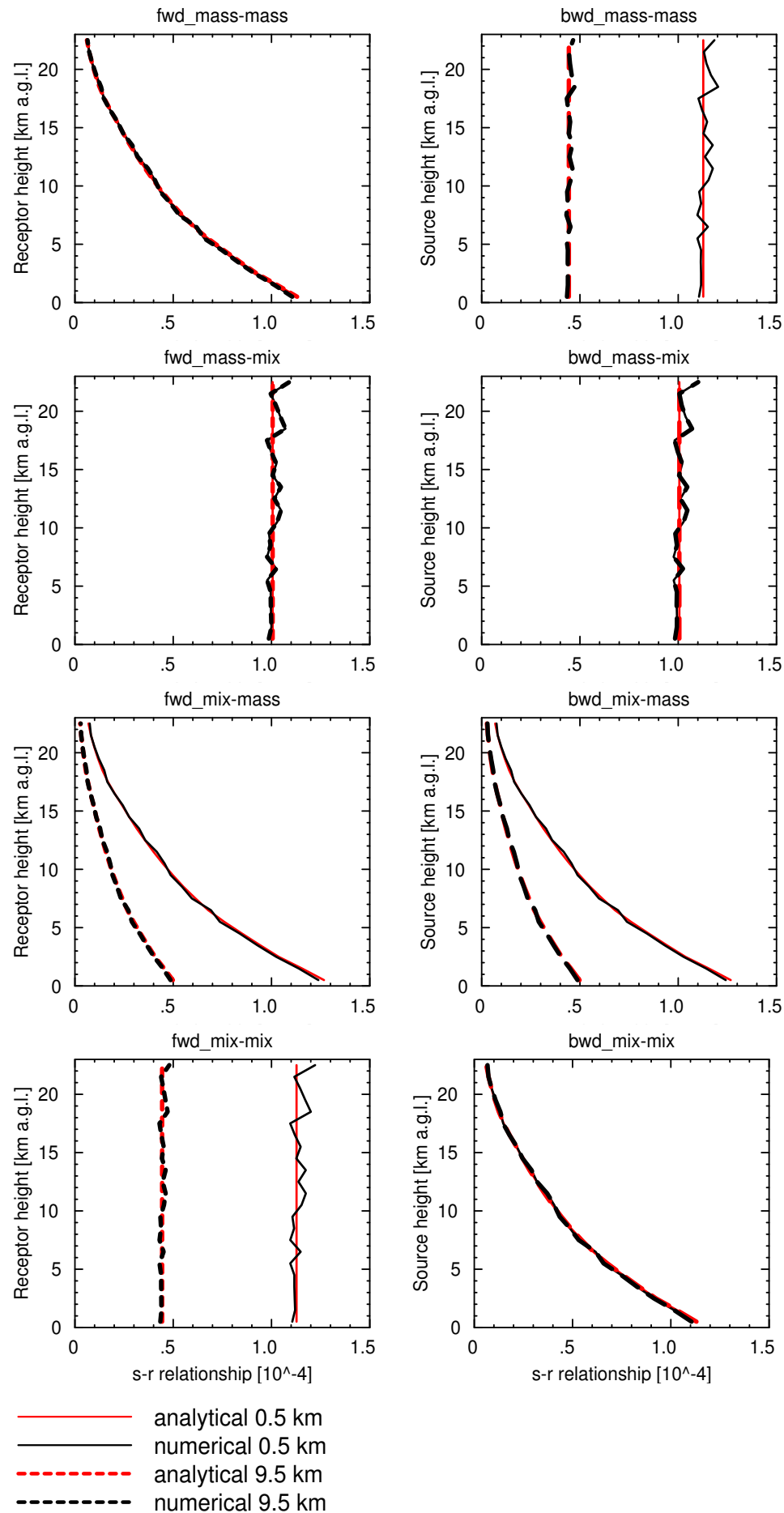

Fig. 3. Analytical (red lines) and numerical (black lines) s-r relationships for idealised convection tests in a $1 \mathrm{~m}^{2}$ air column. Units are according to Table 1, instantaneous local source. The left column of graphs shows forward simulations with s-r relationships as a function of the receptor height for two different source heights (thin solid: $0.5 \mathrm{~km}$, heavy broken: $9.5 \mathrm{~km}$ ). The right column shows backward simulations with $\mathrm{s}-\mathrm{r}$ relationships as a function of the source height for two different receptor heights (colours correspondingly). Forward and backward calculations should agree for these two specific heights. 


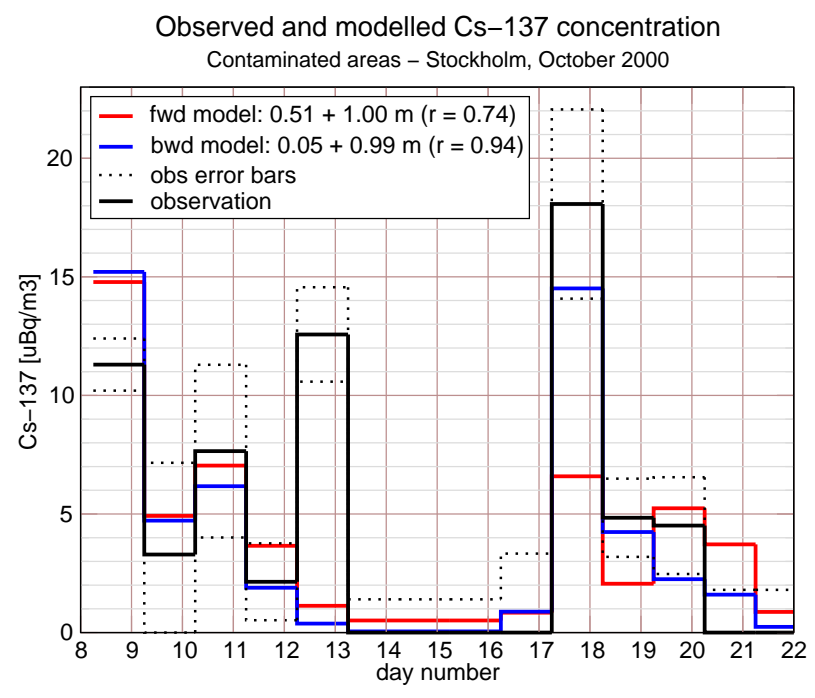

Observed and modelled Cs-137 concentration Chernobyl - Stockholm, October 2000

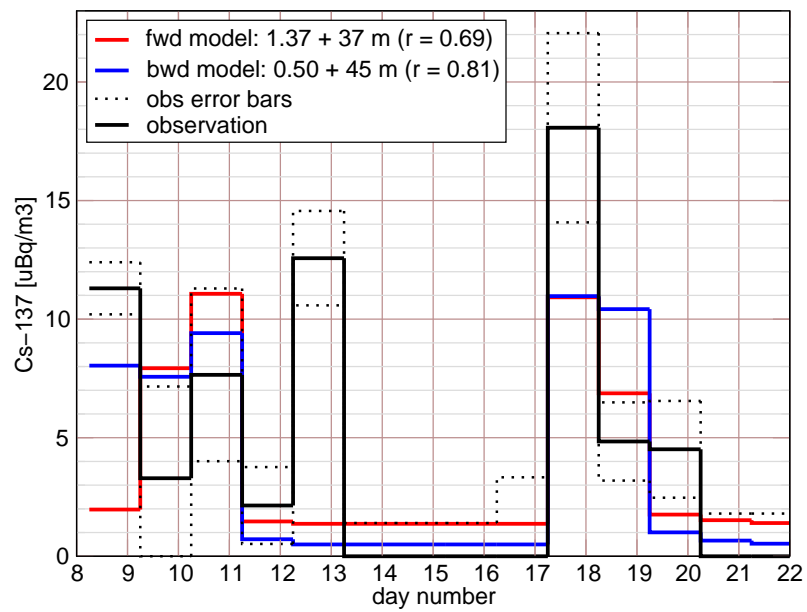

Fig. 4. Time series of the observed (with error margins) and modelled concentration of Cs-137 at Stockholm for a part of October 2000. The model is a simple regression between observations and $\mathrm{s}-\mathrm{r}$ relationship, calculated in either forward or backward mode, between Stockholm and the Chernobyl area. The regression formulae and resulting correlation coefficients are given in the legend; both are determined without the measurement of October 13th (outlier). The top panel is for a source covering the contaminated areas in Ukraine, Belorus and Russia as indicated in Fig. 5, the bottom panel for a source area limited to the grid element containing the Chernobly power plant $\left(0.5^{\circ}\right.$ size $)$.

method, but also as a test for the multipliers given in Table 1, because of the strong differences in ambient pressure. We don't compare forward and backward calculations directly, but rather we compare both of them with the analytical solution. This way of presentation illustrates also the different nature of forward (source-oriented) and backward (receptororiented) calculations. Each forward calculation gives the $s-r$ relationship for one source and the whole profile of receptors, whereas each backward calculation is for one receptor and the whole source profile. Two calculations were performed for each mode, with a low-level and a high-level release. If we denote the source height by $z$ and the receptor height by $\zeta$, and the release in mass units by $\mu$ and in mixing ratio units by $\nu$, the analytical solutions for the $s-r$ relationships read:

$$
\begin{array}{ll}
\text { mass - mass: } & \frac{\partial c(z, \zeta)}{\partial \mu}=\frac{\rho(\zeta)}{M_{A}} \\
\text { mass - mix: } & \frac{\partial \chi(z, \zeta)}{\partial \mu}=\frac{1}{M_{A}} \\
\text { mix - mass: } & \frac{\partial c(z, \zeta)}{\partial v}=\frac{\rho(z) \rho(\zeta)}{M_{A}} \\
\text { mix - mix: } & \frac{\partial \chi(z, \zeta)}{\partial v}=\frac{\rho(z)}{M_{A}}
\end{array}
$$

where $c$ and $\chi$ are the mass concentration and the mass mixing ratio as previously, and $M_{A}$ is the total mass of the air within the column that is being completely mixed. The short source and receptor unit characterisations from Table 1 have been included with each equation for convenience. For a column of $1 \mathrm{~m}^{2}$,

$M_{A}=\left(p_{\text {bottom }}-p_{\text {top }}\right) g^{-1}$

where $p$ is the atmospheric pressure and $g$ is the gravity acceleration.

The test results are shown in Fig. 3. Apart from minor fluctuations which are caused by the stochastic redistribution of the particles by the convection scheme, analytical and numerical $s-r$ relationships agree. It is left to the reader to reflect about the different shapes of the curves and how they are related to the forward or backward calculation modes and the different units for source and receptor.

\section{Application examples}

The new method has already been applied in a study related to the global radionuclide measurement network established as a part of the monitoring system for the Comprehensive Nuclear Test Ban Treaty (Seibert and Frank, 2001), and for the interpretation of aircraft measurements of long-range pollutant transport in the free atmosphere (Stohl et al., 2002).

In addition, a small example shall be presented here. Stations monitoring ambient radioactivity in Europe occasionally measure elevated levels of ${ }^{137} \mathrm{Cs}$ since the Chernobyl disaster. It appears likely that this radioactive material comes 
from resuspension in heavily contaminated areas. We have done some calculations to test this hypothesis for the ${ }^{137} \mathrm{Cs}$ monitoring data of October 2000 in Stockholm, Sweden. Backward as well as forward s-r relationships have been calculated for these measurements as receptors. As the source, we considered either the areas in Ukraine, Belorus and Russia contaminated with more than $100 \mathrm{kBq} \mathrm{m}^{-2}$ according to European Communities et al. (2001), or the single $0.5^{\circ}$ grid cell containing the Chernobyl power plant. Wet and dry deposition were considered in the simulations which are based on ECMWF analyses and - for precipitation and better temporal resolution - short-range forecasts, with $1^{\circ}$ horizontal and $3 \mathrm{~h}$ temporal resolution. The simulations were carried out for transport times up to 7 days. While the receptors have a resolution of 1 to 3 days (depending on the filter change scheme), the source has been temporally integrated over the whole $7 \mathrm{~d}$ transport time. As receptor, a $1^{\circ} \times 1^{\circ}$ grid mesh was used, and the height of both source and receptor grid boxes was $300 \mathrm{~m}$. Simulations were carried out with 500000 particles.

Figure 4 (top) shows that both forward and backward simulations reproduce the major ups and downs of the observations. Strong inhomogeneities of the contamination within the source areas, potential variations in resuspension coefficients and the measurement error (approximated as the minimum detectable activity, varying between 2 and $7 \mu \mathrm{Bq} \mathrm{m}^{-3}$ ) limit the achievable agreement. On some days, there is a considerable difference between forward and backward simulations which we would attribute to general modelling errors, mainly due to interpolation. The sample of October 12/13 is an outlier. It might be caused by caesium picked up in an area northwest of Stockholm (Fig. 5, bottom), at a distance of less than $100 \mathrm{~km}$, contaminated with values in the $40-100 \mathrm{kBq} \mathrm{m}^{-2}$ range (European Communities et al., 2001). Leaving this case out, the model based on the backward simulation results in a correlation coefficient of 0.94 and the expected direct proportionality between $\mathrm{s}-\mathrm{r}$ relationship and concentration (regression constant 0.05). Interestingly, the model based on the forward simulation performs worse (correlation coefficient of 0.74 ).

If only the area immediately around the reactor is considered as potential source (Fig. 4, bottom), the correlation coefficient drops for both the forward and the backward simulation, indicating that it is indeed more likely that the observed caesium originates from resuspension than from ventings of the sarcophagus in which the reactor has been enclosed. Replacing the larger source area with the small one so that both source and receptor are small, the correlation coefficient for the backward model drops much stronger than for the forward model, and the difference between forward an backward models is reduced (especially if considered in terms of explained variance). This could be a hint that a small release area for the computational particles combined with a large receptor area (backward simulation for contaminated areas) is

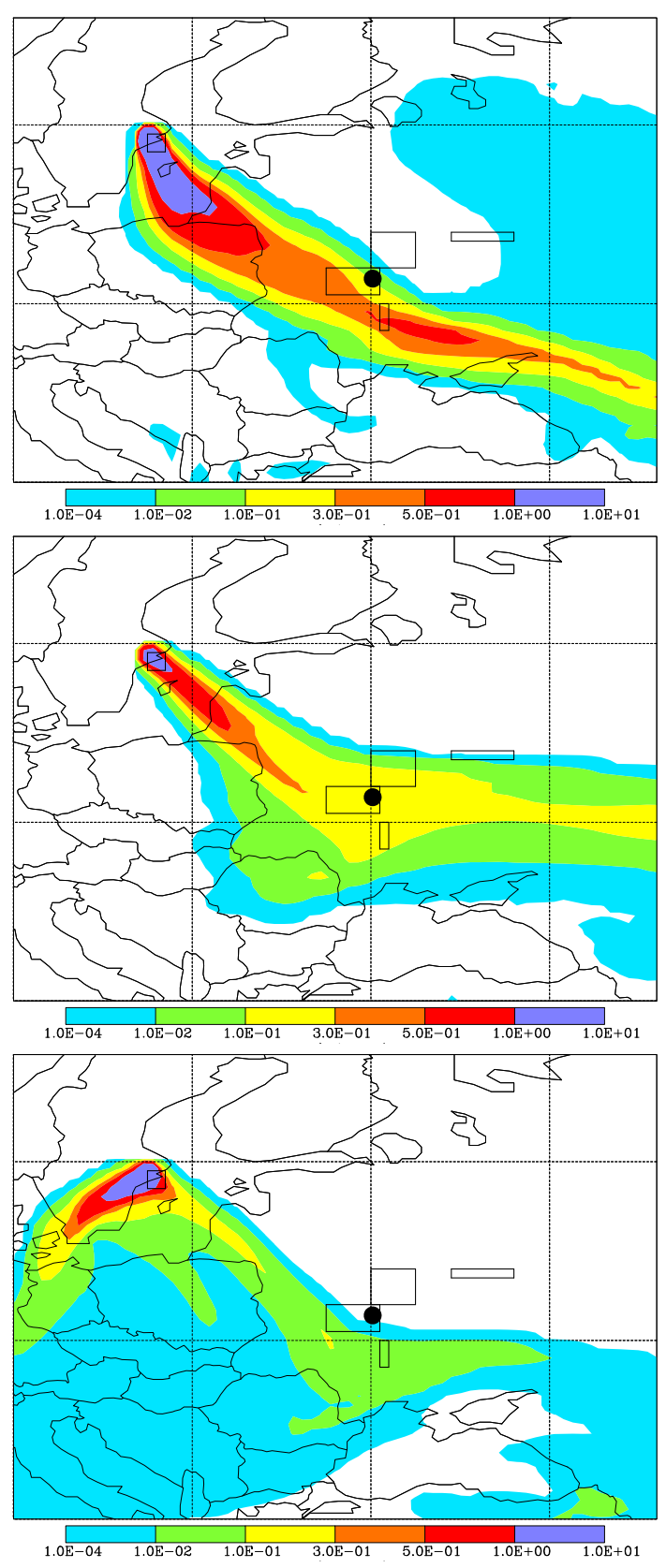

Fig. 5. Some examples of s-r relationships for the receptor at Stockholm as determined in the backward mode for different measurement periods. The s-r relationship is for an area source and has units of $\mathrm{s} \mathrm{m}^{-1}$. The contaminated areas around Chernobyl considered for the evaluation in Fig. 4 are indicated by their perimeters and the Chernobyl power plant site by a black dot. The receptor at Stockholm is marked by a square. Political borders are those of 1986. A geographical grid with a mesh size of $10^{\circ}$ has been overlaid; the southwestern corner is at $40^{\circ} \mathrm{N}, 10^{\circ} \mathrm{E}$. The figures refer to the sampling periods as follows: top: ending on 9 October, middle: 11 October, bottom: 13 October (the outlier). 
less susceptible to numerical errors than vice versa (forward simulation for contaminated areas).

\section{Outlook}

The method introduced in this paper has the potential to supersede back trajectories for the quantitative interpretation of atmospheric trace substance measurements. For more qualitative interpretation, LPDM backward calculations with a trajectory-like compressed output as suggested by Stohl et al. (2002) can be recommended. Our vision is that a background monitoring station would be associated with an archive of the s-r data for each measurement. To keep the archive at a reasonable size, the source vector would probably be gridded only in space but not in time, with one or a few layers vertically and a grid resolution which is finer near the station and coarser at longer distances. Together with a small software tool and an emission data base, the modelled contributions of selectable source regions to the station could be quickly calculated and compared to measurements. Deviations could be analysed in various ways, including inverse modelling (see, e.g. Seibert, 1999). A map of the (2-d or 3-d) s-r relationship (directly or multiplied with emissions) could be termed a "footprint" of that station in analogy to the micro-meteorological flux "footprints". We admit, however, that this wording is not ideal as, rather, emissions leave a kind of "footprint" in the atmosphere, and not vice versa. We suggest an alternative term: the s-r relationship as the "field of view" of a station.

Of course, there are also other methods to calculate such $\mathrm{s}-\mathrm{r}$ climatologies. The advantage of the backward method is obvious for a receptor-oriented view. Compared to an adjoint Eulerian model, the advantage of the LPDM is that there is no initial diffusion due to the release of the adjoint tracer into a finite-size grid cell, which gives the LPDM an advantage as a measurement station acts like a point source in a backward simulation. Furthermore, long-range transport can be simulated more accurately as no artificial numerical diffusion is present. Especially when filaments created by deformation processes become small against the mesh size, Eulerian models reach their limits. There are two drawbacks of the LPDM: it cannot simulate nonlinear chemistry, and for very high dilution ratios, the necessary trade-off between particle number and computational requirements will lead to statistically uncertain results (therefore a coarser grid may be required).

\section{Conclusions}

A method has been derived and introduced with various examples allowing to calculate relationships of substances not undergoing nonlinear chemical changes with a backward- running Lagrangian particle dispersion model. This includes situations with wet and dry deposition or any other firstorder sources and sinks. Receptor-oriented problems, especially related to point or line (aircraft) measurements, can be treated efficiently and relatively accurately by this method.

The method has been tested extensively with the Flexpart (Stohl et al., 1998) model, and useful extensions have been implemented in this model based on experiences gained in these tests. It was also found that many of these tests are generally useful for testing model quality. Differences between forward and backward simulations in realistic conditions may be a useful tool to obtain one estimate of model errors, which is quite important for a subsequent inversion to derive sources.

Acknowledgements. This work was supported by the Austrian Science Fund (FWF) through project P12595 "Inverse modelling of atmospheric trace constituents with Lagrangian models on the European scale", and it is a contribution to the EUROTRAC-2 subprojects GLOREAM and GENEMIS-2. A part of the manuscript was written during a stay of the first author at the Canadian Meteorological Centre (CMC), Montréal, Canada; the support of the Meteorological Service of Canada and the warm reception by the colleagues at $\mathrm{CMC}$ is gratefully acknowledged. The continuous co-operation with A. Stohl (Technical University of Munich, Germany), main author of Flexpart, was important for the integration of the backward calculation mode into this model and provided additional motivation. His contributions to discussions helped to clarify a number of issues in the development of the methodology. Meteorological fields for test calculations were provided by the ECMWF in the frame of the Special Project "SPATMOT". Radionuclide monitoring data from Stockholm were kindly supplied by the Swedish Defense Research Agency (I. Vintersved).

\section{References}

Elbern, H. and Schmidt, H.: A four-dimensional variational chemistry data assimilation scheme for eulerian chemistry transport modeling. J. Geophys. Res. 104(D15), 18 583-18 598, 1999.

Emanuel, K. A. and Živković-Rothman, M.: Development and evalution of a convection scheme for use in climate models, J. Atmos. Sci. 56, 1766-1782, 1999.

European Communities, Roshydromet, Minchernobyl, and Belhydromet: Atlas of caesium deposition on Europe after the Chernobyl accident, Luxembourg, Office for Official Publication of the EU, ISBN 92-894-1004-3, 2001.

Flesch, T. K., Wilson, J. D., and Lee, E.: Backward-time Lagrangian stochastic dispersion models and their application to estimate gaseous emissions, J. Appl. Meteorol. 34, 1320-1333, 1995.

Giering, R.: Tangent linear and adjoint bigeochemical models, In: Inverse Methods in Global Biogeochemical Cycles, edited by Kasibhatla, P., Heimann, M., Rayner, P., Mahowald, N., Prinn, R. G., and Hartley, D. E., pp. 33-48, AGU Geophysical Monograph Vol. 114, ISBN 0-87590-097-6, Washington, 1999. 
de Haan, P.: On the use of density kernels for concentration estimations within particle and puff dispersion models, Atmos. Environ. 33(13), 2007-2021, 1999.

Hourdin, F. and Issartel, J.-P.: Sub-surface nuclear tests monitoring through the CTBT xenon network, Geophys. Res. Let. 127(15), 2245-2248, 2000.

Kljun, N., Rotach, M. W., and Schmid, H. P.: A 3D backward Lagrangian footprint model for a wide range of boundary layer stratifications, Bound.-Layer Meteorol. 103, 205-226, 2002.

Robertson, L. and Persson, C.: On the application of fourdimensional data assimilation of air pollution data using the ajoint technique, In: Air Pollution Modeling and its Application, edited by van Dop, H. and Kallos, G., Vol. IX, pp. 365-373, Penum Press, New York, 1992.

Schmid, H. P.: Footprint modeling for vegetation atmosphere exchange studies, Agric. Forest Meteor. 24, 199-202, 2002.

Seibert, P.: Inverse modelling of sulfur emissions in Europe based on trajectories, In: Inverse Methods in Global Biogeochemical Cycles, edited by Kasibhatla, P., Heimann, M., Rayner, P., Mahowald, N., Prinn,R. G., and Hartley, D. E., pp. 147-154, AGU Geophysical Monograph Vol. 114, ISBN 0-87590-097-6, 1999.

Seibert, P.: Inverse modelling with a Lagrangian particle dispersion model: application to point releases over limited time intervals. In: Air Pollution Modeling and its Application XIV, edited by Schiermeier, F. and Gryning, S.-E., pp. 381-390, Kluwer Academic Publ., 2001.

Seibert, P. and Frank, A.: Backward atmospheric transport and dispersion modelling with FLEXPART in the context of evaluation of atmospheric transport modelling tools at CTBTO/PTS. In: Evaluation of Atmospheric Transport Modelling Tools Used at the Provisional Technical Secretariat, Annex 6., 8 pp., 2001
Seibert, P., Krüger, B., and Frank, A.: Parametrisation of convective mixing in a Lagrangian particle dispersion model. In: Proceedings of the 5th GLOREAM Workshop, Wengen, Switzerland, 2002.

Stohl, A., Eckhardt, S., Forster, C., James, P., Spichtinger, N., and Seibert, P.: A replacement for simple back trajectory calculations in the interpretation of atmospheric trace substance measurements, Atmos. Environ. 36(29), 4635-4648, 2002.

Stohl, A., Hittenberger, M., and Wotawa, G.: Validation of the Lagrangian particle dispersion model Flexpart against large-scale tracer experiment data, Atmos. Environ. 32(24), 4245-4264, 1998.

Stohl, A. and Seibert, P.: The Flexpart particle dispersion model, Version 5.0, User Guide, online at http://www.forst.tu-muenchen.de/EXT/LST/METEO/stohl/ flexpart/flexpart50.pdf, 76 pp., 2002.

Thomson, D. J.: Criteria for the selection of stochastic models of particle trajectories in turbulent flow, Journal of Fluid Mechanics 180, 520-556, 1987.

Uliasz, M.: Application of the perturbation theorie to the sensitivity analysis of an air pollution model, Z. Meteorol. 33(6), 355-362, 1983.

Uliasz, M. and Pielke, R. A.: Receptor-oriented dispersion modeling: extension to nonlinear pollution chemistry, In: Air Pollution Modeling and its Application, Vol. IX, pp. 171-178, 1992. 